\title{
Cellular and Molecular Life Sciences
}

\section{The linker region of breast cancer resistance protein ABCG2 is critical for coupling of ATP-dependent drug transport \\ --Manuscript Draft--}

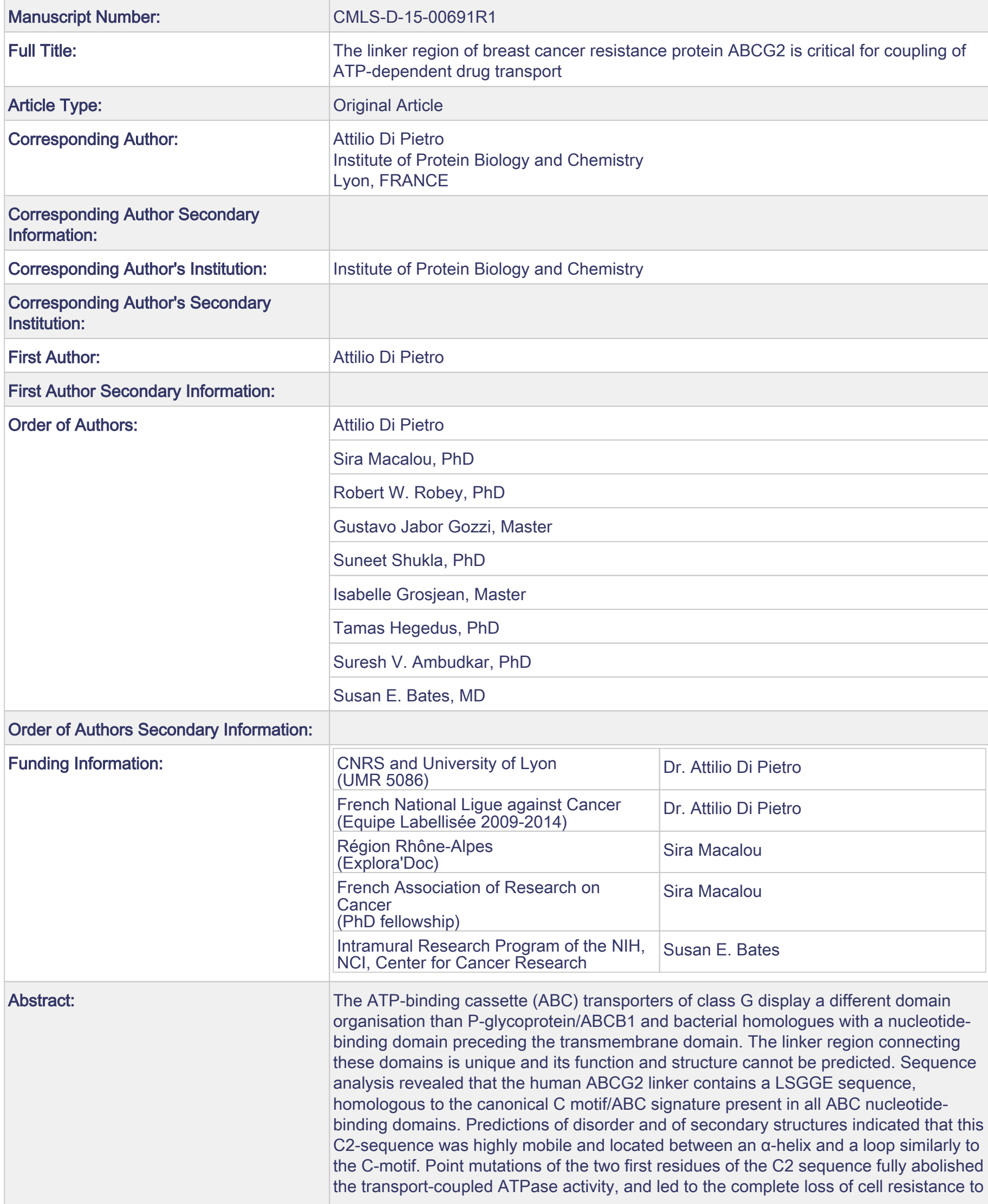


mitoxantrone. The interaction with potent, selective and non-competitive, ABCG2 inhibitors was also significantly altered upon mutation. These results suggest an important mechanistic role for the C2-sequence of the ABCG2 linker region in ATP binding and/or hydrolysis coupled to drug efflux.

Response to Reviewers:

See attachment entitled "Detailed answers to reviewer's comments" 


\section{The linker region of breast cancer resistance protein ABCG2 is critical for coupling of ATP-dependent drug transport}

S. Macalou, R. W. Robey, G. Jabor Gozzi, S. Shukla, I. Grosjean, T. Hegedus, S. V. Ambudkar, S. E. Bates, A. Di Pietro

S. Macalou, G. Jabor Gozzi,A. Di Pietro ( $\triangle)$

Equipe Labellisée Ligue 2014, BMSSI, UMR 5086 CNRS-Université Lyon 1, IBCP, 69007 Lyon, France

e-mail: a.dipietro@ibcp.fr

R. W. Robey, S. E. Bates

Medical Oncology Branch, Center for Cancer Research, National Cancer Institute, National Institutes of Health, Bethesda, MD 20892, USA

S. Shukla, S. V. Ambudkar

Laboratory of Cell Biology, Center for Cancer Research, National Cancer Institute, National Institutes of Health, Bethesda, MD 20892, USA

\section{Grosjean}

CelluloNet biobank BB-0033-00072 facility of UMS3444/US8/SFR Biosciences, IBCP, 69007 Lyon, France

\section{T. Hegedus}

MTA-SE Molecular Biophysics Research Group, Hungarian Academy of Sciences and Department of Biophysics and Radiation Biology, Semmelweis University, Budapest, Hungary.

Running title: Linker region of human ABCG2

Keywords: $A B C$ transporter, breast cancer resistance protein/ABCG2, ATP hydrolysis, $C$ motif/ABC signature, drug efflux coupling, specific sequence. 


\section{Abstract}

The ATP-binding cassette (ABC) transporters of class $\mathrm{G}$ display a different domain organisation than P-glycoprotein/ABCB1 and bacterial homologues with a nucleotidebinding domain preceding the transmembrane domain. The linker region connecting these domains is unique and its function and structure cannot be predicted. Sequence analysis revealed that the human ABCG2 linker contains a LSGGE sequence, homologous to the canonical $C$-motif/ABC signature present in all $A B C$ nucleotide-binding domains. Predictions of disorder and of secondary structures indicated that this $\mathrm{C} 2$-sequence was highly mobile and located between an $\alpha$-helix and a loop similarly to the C-motif. Point mutations of the two first residues of the C2-sequence fully abolished the transport-coupled ATPase activity, and led to the complete loss of cell resistance to mitoxantrone. The interaction with potent, selective and non-competitive, ABCG2 inhibitors was also significantly altered upon mutation. These results suggest an important mechanistic role for the C2-sequence of the ABCG2 linker region in ATP binding and/or hydrolysis coupled to drug efflux. 


\section{Introduction}

The ABCG2 transporter was discovered simultaneously in three different groups; it was called $A B C P$ for its abundance in placenta [1], BCRP as a breast cancer resistance protein [2] and MXR for its induced resistance to mitoxantrone [3]. Its overexpression in cancer cells was identified to strongly contribute to multidrug resistance, similarly to previously discovered P-glycoprotein/ABCB1 [4, 5] and MRP1/ABCC1 [6]. ABCG2 also plays an essential physiological control within barriers protecting sensitive organs [7, 8] as well as in stem cells of which it is considered to be a marker [9].

All members of the ATP-binding cassette $(A B C)$ superfamily are constituted of cytosolic nucleotide-binding domains (NBDs), responsible for ATP binding and hydrolysis, and two transmembrane domains (TMDs) generally comprising 6 $\alpha$-helical spans ensuring substrate transport across the membrane. These four domains are fused through linkers into a single TMD1-NBD1-TMD2-NBD2 polypeptide, as a "full-transporter" in P-glycoprotein (ABCB1) and MRP1 (which also contains an additional N-terminal TMD0 domain). In contrast to ABCB1 and MRP1, ABCG2 is a "half-transporter", with a single NBD fused to a single TMD, and therefore requires dimerization, at a minimum, to form a functional unit. Its NBD-TMD domain arrangement is different; as a consequence, the linker connecting both domains is unique and its structure cannot be modeled using a template, such as the crystal structures of P-glycoproteins from mice [10] or Caenorhabditis elegans [11], or of a bacterial homologue [12].

All NBDs of the ABC transporters contain several ATP-binding motifs: in addition to the Walker-A and Walker-B motifs also present in other ATPases, a conserved specific sequence LSGGQ, followed by three basic residues, is called "Cmotif" or "C-loop" and is considered to be an "ABC signature". Its critical function in 
ATP hydrolysis and in coupled substrate transport was demonstrated by site-directed mutagenesis in various transporters such as P-glycoprotein [13, 14], MRP1 [15], TAP $[16,17]$ and MalK [18]. The crystal structure of HlyB precisely identified the interactions with different moieties (adenine, ribose and $\gamma$-phosphate) of bound ATP, and additionally showed protein-protein interactions with the Walker-A motif of the other NBD [19]. The C-motif is therefore contributing to NBD dimerization, which is required for ATP hydrolysis and its coupling to the transport process by two types of direct interactions: (1) with ATP, mainly bound to the other NBD through Walker-A, Walker-B and other motifs, and (2) with the Walker-A motif of the other NBD.

In the present work, an additional C-motif LSGGE, called the C2-sequence, was identified at positions 352-356, within the poorly known linker region of human ABCG2. The aim was therefore to look at its conservation among ABCGs and species, and a potential function. The results show that the C2-sequence is predicted to be quite mobile and structurally similar to the C-motif; point mutations fully altered ABCG2-mediated coupled ATPase activity and cellular multidrug resistance, suggesting a critical role in coupling between ATP hydrolysis and drug efflux.

\section{Materials and Methods}

Sequence alignment and structure predictions

Sequences were downloaded from UniProt [20]. Sequence alignments were generated using ClustalW using default parameters [21], analyzed and displayed in Cinema5 (http://aig.cs.man.ac.uk/research/utopia/cinema/cinema.php). Sequences were queried for the $A B C$ signature pattern (ProSite; http://prosite.expasy.org/PDOC00185) employing the preg software from EMBOSS (The European Molecular Biology Open Software Suite) [22]. Two conceptually different algorithms were used to predict disorder tendency with default parameters 
including DISOPRED2 [23] and IUPRED [24]. Secondary structure prediction was done employing also different predictors: Prof (http://www.aber.ac.uk/ phiwww/prof/), Jnet [25] and PSI [26].

\section{Compounds}

Commercial reagents were of the highest available purity grade: mitoxantrone $(\geq 97 \%)$ and Ko143 ( $\geq 98 \%)$ were purchased from Sigma-Aldrich (France), and nilotinib from Selleck Chemicals (Boston, MA). Chromone 1 was obtained as previously described [27]. The 5D3 monoclonal antibody was purchased from eBioscience and BXP-21 antibody from Alexis Biochemicals.

\section{Generation of Mutants}

The L352A, S353A, G354A, G355A and E356A point mutants were generated by site-directed mutagenesis in a pcDNA3.1 vector (Invitrogen) carrying the full length $A B C G 2$ cDNA, kindly provided by the laboratory of Dr. Douglas Ross. Sited-directed mutagenesis was carried out using the "QuickChange® Site-Directed Mutagenesis" kit (Stratagene). The plasmid containing ABCG2 was amplified by PCR from primers containing the desired mutation. The full-length sequences of all mutant constructs were tested for correctness by sequencing. The designed primer sequences are presented in the following table, where the mutated nucleotides are in bold letters.

Primers Sequences

L352A-Fw ACAAAAGCTGAATTACATCAAGCTTCCGGGGGTGAGAAG

L352A-Rv CTTCTCACCCCCGGAAGCTTGATGTAATTCAGCTTTTGT

S353A-Fw AAAGCTGAATTACATCAACTTGCCGGGGGTGAGAAGAAG

S353A-Rv CTTCTTCTCACCCCCGGCAAGTTGATGTAATTCAGCTTT

G354A-Fw CCTGAATTACATCAACTTTCCGCGGGTGAGAAGAAGAAG

G354A-Rv CTTCTTCTTCTCACCCGCGGAAAGTTGATGTAATTCAGG 


\section{G355A-Fw TTACATCAACTTTCCGGGGCTGAGAAGAAGAAGAAGATC \\ G355A-Rv GATCTTCTTCTTCTTCTCAGCCCCGGAAAGTTGATGTAA \\ E356A-Fw TTACATCAACTTTCCGGGGGTGCGAAGAAGAAGAAGATC \\ E356A-Rv GATCTTCTTCTTCTTCGCACCCCCGGAAAGTTGATGTAA}

\section{Cell cultures}

Human Embryonic 293 cell line (CelluloNet $n^{\circ 109)}$ was transfected (Nucleofector technique, solution 5, programme A23, LONZA) with either ABCG2 (HEK293ABCG2, also called "293BCRP3, CelluloNet $\mathrm{n}^{\circ} 234$ ") or the empty vector (HEK293pcDNA3) or ABCG2 mutants. Stable polyclonal cell lines were then selected with $0.75 \mathrm{mg} / \mathrm{ml} \mathrm{G} 418$, and cell lines were cloned. They were maintained in high-glucose DMEM supplemented with $10 \%$ fetal bovine serum (FBS), $1 \%$ penicillin/streptomycin at $37{ }^{\circ} \mathrm{C}, 5 \% \quad \mathrm{CO}_{2}$ under controlled humidity. The cell culture medium was supplemented with $0.75 \mathrm{mg} / \mathrm{mL}$ G418.

\section{$5 D 3$ binding}

In studies with the anti-ABCG2 antibody 5D3, cells were incubated in $2 \%$ BSA/DPBS (Dulbecco's Phosphate Buffered Saline) with either phycoerythrin-labeled negative control antibody (IgG2b) or phycoerythrin-labeled 5D3 antibody (both from eBioscience, San Diego, CA), used in excess according to the manufacturer's instructions, washed with DPBS, and subsequently analyzed. Surface expression of ABCG2 was calculated as the difference in mean channel numbers between the 5D3 histogram and the negative-control antibody histogram. Samples were analyzed on a FACSort flow cytometer (Becton Dickinson, San Jose, CA). Phycoerythrin fluorescence was detected with a $488 \mathrm{~nm}$ argon laser and a $585 \mathrm{~nm}$ bandpass filter. At least 10,000 events were collected for each flow cytometry experiment. By gating 
on forward versus side scatter, debris was eliminated, and dead cells were excluded based on propidium iodide staining.

\section{Mitoxantrone efflux and inhibition by characteristic inhibitors}

HEK-293 cells expressing wild-type or mutated ABCG2 were trypsinized and incubated for 30 min with $20 \mu \mathrm{M}$ mitoxantrone in the presence or absence of $10 \mu \mathrm{M}$ of the ABCG2 inhibitor fumitremorgin $C$ (FTC). Cells were then washed and incubated for $1 \mathrm{~h}$ in mitoxantrone-free medium continuing with or without FTC. Mitoxantrone efflux was calculated by subtracting the mean fluorescence of the cells incubated without FTC from the mean fluorescence of the cells incubated with FTC as previously described [28].

\section{Cell growth resistance to mitoxantrone}

HEK-293 cells expressing wild-type or mutated ABCG2, as well as control cells were seeded at a density of $10^{4}$ cells/well into 96-well culture plates, incubated overnight, and treated with mitoxantrone (up to $1 \mu \mathrm{M}$ ) for $72 \mathrm{~h}$. To assess viability, the cells were exposed to $0.5 \mathrm{mg} / \mathrm{mL}$ MTT and incubated for $4 \mathrm{~h}$ at $37^{\circ} \mathrm{C}$ [29]. The culture medium was discarded, and $100 \mu \mathrm{L}$ of a DMSO/ethanol (1:1) solution was added into each well and mixed by gently shaking for $10 \mathrm{~min}$. Absorbance was measured at 570 $\mathrm{nm}$ using a microplate reader, and the value measured at $690 \mathrm{~nm}$ was subtracted. Data are the mean \pm SD of at least three independent experiments.

\section{ATPase activity assay}

Beryllium-fluoride-sensitive ATPase activity was assayed using crude membranes (10 $\mu \mathrm{g}$ protein/tube) of HEK293 cells expressing WT and mutant ABCG2 as described previously [30]. Briefly crude membranes isolated from HEK cells 
expressing ABCG2 (1 mg of protein $/ \mathrm{ml}$ ) were incubated at $37^{\circ} \mathrm{C}$ in ATPase assay buffer (50 mM KCl, $5 \mathrm{mM}$ sodium azide, $2 \mathrm{mM}$ EGTA, $1 \mathrm{mM}$ ouabain, $10 \mathrm{mM} \mathrm{MgCl}$, $2 \mathrm{mM}$ dithiothreitol, and $50 \mathrm{mM}$ Tris-MES, $\mathrm{pH}$ 6.8) in the presence or absence of indicated substrates for $5 \mathrm{~min}$. The ATPase reaction was started by the addition of 5 mM ATP and was terminated by the addition of $0.1 \mathrm{ml}$ SDS solution after $20 \mathrm{~min}$. The amount of inorganic phosphate released was quantified by a colorimetric reaction as described previously [30].

\section{Results}

\section{Identification of a specific C2-sequence in human ABCG2}

Sequence alignment of the different human ABCG transporters indicated the following consensus for the canonical $A B C$ signature/C-motif, L/V-S-G-G-E/Q followed by R-K/R-R (Fig. 1A), except for some degenerescence in ABCG5 which is fuctionally active as an heterodimer with ABCG8. The ABCG2 sequence located at positions 188-192 was V-S-G-G-E. Quite interestingly, a very similar additional sequence, called hereafter C2-sequence (L-S-G-G-E, followed by K-K-K), was found exclusively in human ABCG2, at positions 352-356 (Fig. 1B). It obeyed the consensus sequence illustrated here above for human ABCG members, and known to be common to all ABC transporters; it could therefore be defined as an extra, noncanonical, C-motif. Sequence alignment of human ABCG2 with ABCG2 homologues from different species indicated in Fig. 1C that this non-canonical human C2sequence was well conserved in mammals (elephant, dog, horses, goat, bovines, ovines, primates and rodents), partly in avian organisms (chicken, turkey) and in amphibia, but not in fishes, protozoan parasites and plants. Interestingly, no C2sequence was found in yeast MDR full-transporters, with similar domain organisation 
as ABCG2, such as Saccharomyces cerevisiae Pdr5p or Candida albicans Cdr1p (not shown).

Secondary structure predictions indicate the C2-sequence located at the border of an $\alpha$-helix and a region without stable secondary structure similarly as the canonical C-motif. Moreover, the latter segment around a.a. 360 is predicted to be the most disordered part of the 301-396 linker region when using the DISPORED2 program, and one among the regions with higher disorder tendency determined by the IUPred program (Fig. 2). The coherence of the secondary structure and disorder predictions employing different algorithms strengthens the hypothesis that the C2sequence can be embedded into a similar structural environment as the canonical Cmotif.

Slight alterations of C2-sequence mutations on cell surface expression and activity of ABCG2

Single point mutation into alanine of each of the five residues of the $\mathrm{C} 2$-sequence (L352-S353-G354-G355-E356) induced variable consequences on the total ABCG2 expression monitored by Western blotting with the BXP-21 ABCG2-specific antibody: from at least 2-fold decrease versus the wild-type protein for L352A, S353A and E356A mutants, to no apparent effect for the G354A and G355A mutants (not shown here). However, the plasma membrane expression of ABCG2, as monitored with the 5D3 antibody recognizing the ABCG2 extracellular loop 3, was hardly modified except for the S353A mutant exhibiting a decrease of $13 \%$ (Fig. 3A-B). The correct trafficking to plasma membrane was visualized and confirmed by confocal microscopy (Fig. 3C). Mitoxantrone efflux was measured by flow cytometry as differential intracellular accumulation (Fig. 4A-B) and was also corrected for the small differences observed in membrane expression: the two L352A and S353A mutants 
showed a moderate decrease of $23-32 \%$ in activity when compared to that of the wild-type ABCG2 (Fig. 4C). Symmetrically, a positive control with the gain-of-function R482G mutant showed a marked increase in mitoxantrone efflux: about $45 \%$ versus wild-type ABCG2, and 90-115\% versus the two L352A and S353A mutants.

\section{C2-mutants exhibit a markedly decreased resistance to mitoxantrone}

The consequences of point mutations were much more pronounced in $72 \mathrm{~h}$ long cytotoxicity assays, performed in the presence of mitoxantrone and quantified by a cell survival MTT test. A complete loss of resistance to mitoxantrone cytotoxicity was observed with both L352A (Fig. 5A) and S353A (Fig. 5B) mutants, both of which reached sensitivity levels similar to that of the ABCG2-negative HEK-293 control cells transfected with the empty pcDNA3.1 vector. A lower, but significant, chemosensitization was observed with the G354A (Fig. 5C) and E356A (Fig. 5D) mutants.

\section{Alterations of basal and drug-stimulated ATPase activities}

The beryllium-fluoride-sensitive ATPase activity of membranes, prepared from resistant ABCG2-overexpressing HEK-293 cells, was altered upon point mutations, both in the absence or presence of drug substrate (Fig. 6). While the basal ATP hydrolysis (14.6 nmol Pi/min.mg protein) was decreased (up to $30 \%$ ) for the S353A mutant, L352A and S353A mutants showed at least a 3-fold decrease in the $\mathrm{Km}$ values for ATP hydrolysis (Fig. 6A). More strikingly, the stimulation of basal ATPase activity in the wild-type protein by transport substrates such as nilotinib [31] was completely abolished in L352A and S353A mutants (Fig. 6B), whereas nilotinib still stimulated the ATPase activity of G354A and E356A mutants. 
Alterations of the interaction with different types of drug-efflux inhibitors

The point-mutation effect on the efficiency of different types of inhibitors was studied with S353A, the most altered mutant, in comparison with wild-type ABCG2 (Fig. 7). The inhibition of mitoxantrone efflux by Ko143, an ABCG2-selective and highlypotent inhibitor [32] known to also inhibit the basal ATPase activity [33] and not to be transported, was found here to be strongly impaired in this mutant. The $\mathrm{EC}_{50}$ of Ko143 [34) and chromone 1 [27], which are known to inhibit ABCG2-mediated mitoxantrone efflux, was increased from 0.06 to $0.26 \mu \mathrm{M}$ (4.3-fold increase) and from 0.11 to $0.39 \mu \mathrm{M}$ (3.5-fold increase), respectively (Fig. 7A and 7B). By contrast, no significant alteration in the $\mathrm{EC}_{50}$ values was observed for the inhibition of activity by nilotinib, a competitive inhibitor [35] known to be transported by ABCG2 [31] (Fig. $7 C)$.

\section{Discussion}

This paper demonstrates the functional role of a selective sequence of human ABCG2, identified as a potential extra ATP-binding motif, and its unique implication in the coupling mechanism between ATP hydrolysis and drug-efflux activity.

\section{The ABCG2-selective C2-sequence as an additional C-motif?}

Three different approaches have identified the C2-sequence LSGGE, at 352-356 positions of human $A B C G 2$, as a possible nucleotide-binding motif. First, sequence alignment unambiguously showed complete agreement with the consensus sequence of the canonical C-motif/ABC signature found in all NBDs; second, disorder and secondary-structure predictions indicate the C2-sequence to be located between an $\alpha$-helix and a loop similarly to the canonical C-motif; third, the strong 
alterations induced upon point-mutations further confirmed a critical function, associated to both ATPase and drug-efflux activities.

This suggests that the C2-sequence, belonging to the linker connecting NBD and TMD, might be possibly located close to the ATP-binding site of the NBD to directly interact with bound nucleotide. The C2-sequence, at positions 352-356, is distant by about 40 residues from the beginning of TMD, which is similar to the length of the intracellular loops in crystallized bacterial Sav1866 [12] and eukaryotic P-glycoproteins $[10,11]$. However, it not known if the presence of the C2-sequence might compensate the lack of a long intracellular loop, as shown in the human ABCG2 molecular model [36], or if such a multidrug homodimeric half-transporter might require additional interactions than full-transporters such as ABCB1 and ABCC1. It is not either known if the absence of such a C2-sequence in lower eukaryotes might be correlated to differences in the regulation of activity or in structural divergence of these ABCG2 homologs.

\section{A critical function of the C2-sequence}

Single-point mutations have allowed here the identification of two types of dramatic functional alterations. The first type concerned the ATPase activity, for which a decrease in both $V_{\max }$ and $\mathrm{K}_{\mathrm{m}}$ for ATP hydrolysis was observed; this suggested a role of the C2-sequence in either ATP binding or in ADP release, which is known to be often a rate-limiting step of ATP hydrolysis for various ATPases. In addition, the complete abolition of drug-induced stimulated ATP hydrolysis, recognized as coupling ATPase activity, was consistent with the strong catalytic impairment observed upon C-motif point-mutations in various $A B C$ transporters, such as the second glycine in either only P-glycoprotein NBD1 [13] or both MRP1 NBD1 and NBD2 [15], the two serines in both P-glycoprotein NBD1 and NBD2 [14], and either 
single glycine or glutamine in homodimeric MalK [18]. For TAP1/TAP2 heterodimer, it was demonstrated, by point-mutations within both monomers and C-motifs exchange in chimeras, that the strong alterations observed on substrate transport were correlated to changes in ATP hydrolysis [17], and not to ATP binding [16], and that the natural substitutions in degenerated TAP2 (AA versus SG in canonical TAP1) allowed the control of transport activity and coupled ATP hydrolysis [17]. It is not known whether residues of the ABCG2 C2-sequence might additionally establish protein-protein interactions contributing to the closure/dimerization of NBDs as shown for the C-motif within the crystal structure of HlyB [19]. The much stronger consequences induced on coupled versus basal ATPase indicates that both activities obey distinct mechanisms, as previously concluded from different approaches with either ABCG2 [34] or P-glycoprotein [37].

The second type of alteration concerned drug-efflux activity: i) the complete loss of cellular resistance to mitoxantrone cytotoxicity, upon 3-day culturing, appeared to contrast with the only partial decrease of mitoxantrone efflux observed in short-term flow-cytometry experiments. Different hypothetical explanations might be considered, such as time-dependent amplification under cell-culture conditions of the initially moderate alteration of drug efflux, changed $\mathrm{IC}_{50}$ values of mitoxantrone for its target, or mitoxantrone-induced lowered metabolic activity of mutant cells reducing ATP concentration below treshold for basal ATPase activity (?). A marked alteration in substrate transport was also observed upon TAP1/TAP2 mutations, as mentioned above $[16,17]$. Since the same C2-sequence point mutations induced a complete, primary, alteration of coupled ATP hydrolysis, the alterations observed on drug efflux might be considered as consequences, or secondary effects, rather than a direct contribution of mutated residues; ii) the strong alteration of the potent, selective, and noncompetitive Ko143 and chromone 1 inhibitors [34], known to inhibit basal ATPase 
activity, suggested (despite their high hydrophobicity) a rather "cytosolic" inhibitorbinding site, in close proximity to the NBDs where ATP binds, although a distant conformational change cannot be excluded. The interaction of these inhibitors with the selective C2-sequence might, at least partly, explain their ABCG2 selectivity versus the other multidrug $\mathrm{ABC}$ transporters $\mathrm{P}$-glycoprotein/ABCB1 and MRP1/ABCC1.

It may be concluded that the C2-sequence, especially L352 and S353 in human ABCG2, plays a unique role in coupling ATP hydrolysis to drug efflux, and related conformational changes of the transporter. This might constitute a new selective target to antagonize multidrug resistance in ABCG2-overexpressing cancer cells.

\section{Acknowledgments}

Drs. A. Ahmed-Belkacem and C. Gauthier are acknowledged for their help in initiating the studies and performing some experiments, respectively. This work was supported by the CNRS and University Lyon 1 (UMR 5086), and the Ligue Nationale contre le Cancer (Equipe Labellisée Ligue 2014) to A.D.P. S.M. was recipient of fellowships from the Ligue de la Loire contre le Cancer, the Association pour la Recherche sur le Cancer and the Région Rhône-Alpes (Explora'Doc mobility program with S.E.B.). R.W.R., S.S., S.V.A. and S.E.B. were supported by the Intramural Research Program of the NIH, National Cancer Institute, Center for Cancer Research. The authors also thank research funding from OTKA K 111678 and the Bolyai Fellowship of the Hungarian Academy of Sciences to T.H. 


\section{References}

1. Allikmets R, Schriml LM, Hutchinson A, Romano-Spica V, Dean M (1998) A human placenta-specific ATP-binding gene (ABCP) on chromosome 4q22 that is involved in multidrug resistance. Cancer Res 58(23):5337-5339

2. Doyle LA, Yang W, Abruzzo LW, Krogmann T, Gao Y, Rishi AK, Ross DD (1998) A multidrug resistance transporter from human MCF-7 breast cancer cells. Proc Natl Acad Sci USA 95(26):15665-15670

3. Miyake K, Mickley L, Litman T, Zhan Z, Robey R, Cristensen B, Brangi M, Greenberger L, Dean M, Fojo T, Bates SE (1999) Molecular cloning of cDNAs which are highly expressed in mitoxantrone-resistant cells : demonstration of homology to $A B C$ transport genes. Cancer Res 59(1):152-162

4. Juliano RL, Ling V (1976) A surface glycoprotein modulating drug permeability in Chinese hamster ovary cell mutants. Biochim Biophys Acta 455(1):8-13

5. Ueda K, Clark DP, Chen CJ, Roninson IB, Gottesman MM, Pastan I (1987) The human multidrug resistance (mdr1) gene. cDNA cloning and transcription initiation. J Biol Chem 262(2):505-508

6. Cole SP, Bhardwaj G, Gerlach JH, Mackie JE, Grant CE, Almquist KC, Stewart AJ, Kurz EU, Duncan AM, Deeley RG (1992) Overexpression of a transporter gene in a multidrug-resistant human lung cancer cell line. Science 258(5088):1650-1654

7. Jonker JW, Buitlelaar M, Wagenaar E, van de Valk MEA, Scheffer GL, Scheper RJ, Plosch T, Kuipers F, Elferink RP, Rosing H, Beijnen JH, Schinkel AH (2002) The breasr cancer resistance protein protects against a major chlorophyll-derived dietary phototoxin and protoporphyria. Proc Natl Acad Sci USA 99(24):1564915654 
8. Sarkadi B, Homolya L, Szakacs G, Varadi A (2006) Human multidrug resistance $A B C B$ and $A B C G$ transporters: participation in a chemoimmunity defense system. Physiol Rev 86(4):1179-1236

9. Zhou S, Morris JJ, Barnes Y, Lan L, Schuetz JD, Sorrentino BP (2002) Bcrp1 gene expression is required for normal numbers of side population stem cells in mice, and confers relative protection to mitoxantrone in hematopoietic cells in vivo. Proc Natl Acad Sci USA 99(19):12339-12344

10. Aller SG, Yu J, Ward A, Weng Y, Chittaboina S, Zhuo R, Harrell PM, Trinh YT, Zhang Q, Urbatsch IL, Chang G (2009) Structure of P-glycoprotein reveals a molecular basis for ply-specific drug binding. Science 323(5922):1718-1722. doi: $10.1126 /$ science. 1168750

11. Jin MS, Oldham ML, Chen J (2012) Crystal structure of the multidrug transporter P-glycoprotein from Caenorhabditis elegans. Nature 490(7421):566-569. doi: 10.1038/nature. 11448

12. Dawson RJ, Locher KP (2006) Structure of a bacterial multidrug ABC transporter. Nature 443(7108):180-185

13. Bakos E, Klein I, Welker E, Szabo K, Muller M, Sarkadi B, Varadi A (1997) Characterization of the human multidrug resistance protein containing mutations in the ATP-binding cassette signature region. Biochem J 323(Pt 3):777-783

14. Tombline G, Bartholomew L, Gimi K, Tyndall GA, Senior AE (2003) Synergy between conserved $A B C$ signature Ser residues in P-glycoprotein catalysis. J Biol Chem 279(7):5363-5373

15. Szentpetery Z, Kern A, Liliom K, Sarkadi B, Varadi A, Bakos E (2004) The role of the conserved glycines of ATP-binding cassette signature motifs of MRP1 in the communication between the substrate-binding site and the catalytic centers. $J$ Biol Chem 279(40):41670-41678 
16. Hewitt EW, Lehrer PJ (2003) The ABC-transporter signature motif is required for peptide translocation but not peptide binding by TAP. Eur J Immunol 33(2):422427

17. Chen M, Abele R, Tampé R (2004) Functional non-equivalence of ATP-binding cassette signature motifs in the transporter associated with antigen processing (TAP). J Biol Chem 279(44):46073-46081

18. Schmees G, Stein A, Hunke S, Landmesser H, Schneider E (1999) Functional consequences of mutations in the conserved "signature sequence" of the ATPbinding cassette protein MalK. Eur J Biochem 266(2):420-430

19. Zaitseva J, Jenewein S, Jumpertz T, Holland IB, Schmitt L (2005) H662 is the linchpin of ATP hydrolysis in the nucleotide-binding domain of the $A B C$ transporter HlyB. EMBO J 24(11):1901-1910

20. The UniProt Consortium (2015) UniProt: a hub for protein information. Nucleic Acids Res 43: D204-D212

21. Larkin MA, Blackshields G, Brown NP, Chenna R, McGettigan PA, McWilliam H, Valentin F, Wallace IM, Wilm A, Lopez R, Thompson JD, Gibson TJ, Higgins DG (2007) ClustalW and ClustalX version 2. Bioinformatics 23(21): 2947-2948

22. Rice P, Longden I, Bleasby A (2000) EMBOSS: The European Molecular Biology Open Software Suite. Trends in Genetics 16(6):276-277

23. Ward JJ, Sodhi JS, McGuffin LJ, Buxton BF and Jones DT (2004) Prediction and functional analysis of native disorder in proteins from the three kingdoms of life. Journal of Molecular Biology (337):635-645

24. Dosztányi Z, Csizmók V, Tompa P, Simon I (2005) The Pairwise Energy Content Estimated from Amino Acid Composition Discriminates between Folded and Intrinsically Unstructured Proteins. J Mol Biol 347:827-839 
25. Cole C, Barber JD, Barton GJ (2008) The Jpred 3 secondary structure prediction server. Nucleic Acids Research 36:W197-W201

26. Buchan DWA, Minneci F, Nugent TCO, Bryson K, Jones DT (2013) Scalable web services for the PSIPRED Protein Analysis Workbench. Nucleic Acids Research 41:W340-W348

27. Valdameri G, Genoux-Bastide E, Peres E, Gauthier C, Guitton J, Terreux R, Winnischofer SM, Rocha ME, Boumendjel A, Di Pietro A (2012) Substituted chromones as highly potent nontoxic inhibitors, specific for the breast cancer resistance protein. J Med Chem 55(2):966-970. doi: 10.1021/jm201404w

28. Morisaki K, Robey RW, Ozvegy-Laczka C, Honjo Y, Polgar O, Steadman K, Sarkadi B, Bates SE (2005) Single nucleotide polymorphisms modify the transporter activity of ABCG2. Cancer Chemother Pharmacol 56(2):161-172

29. Mosmann T (1983) Rapid colorimetric assay for cellular growth and survival. Application to proliferation and cytotoxicity assays. J Immunol Methods 65(12):55-63

30. Shukla S, Robey RW, Bates SE, Ambudkar SV (2006) The calcium-channel blockers, 1,4-dihydropyridines, are substrates of the multidrug resistancelinkedABC drug transporter, ABCG2. Biochemistry 45(29):8940-8951

31. Shukla S, Skoumbourdis AP, Walsh MJ, Hartz AM, Fung KL, Wu CP, Gottesman MM, Bauer B, Thomas CJ, Ambudkar SV (2011) Synthesis and characterization of a BODIPY conjugate of the BCR-ABL kinase inhibitor Tasigna (nilotinib): evidence for transport of Tasigna and its fluorescent derivative by $A B C$ drug transporters. Mol Pharmaceut. 8(4):1292-1302. doi: 10.1021/mp2001022

32. Allen JD, van Loevezijn A, Lakhai JM, van der Valk M, van Tellingen O, Reid G, Schellens JH, Koomen GJ, Schinkel AH (2002) Potent and specific inhibition of 
the breast cancer resistance protein multidrug transporter in vitro and in mouse intestine by a novel analogue of fumitremorgin C. Mol Cancer Ther 1(6):417-425

33. Ozvegy-Laczka C, Hegedus T, Varady G, Ujhelly O, Schuetz JD, Varadi A, Keri G, Orfi L, Nemet K, Sarkadi B (2004) High-affinity interaction of tyrosine kinase inhibitors with the ABCG2 multidrug transporter. Mol Pharmacol 65(6):1485-1495

34. Winter E, Lecerf-Schmidt F, Jabor Gozzi G, Peres B, Lightbody M, Gauthier C, Ozvegy-Laczka C, Sarkadi B, Creczynski-Pasa TB, Boumendjel A, Di Pietro A (2013) Structure-activity relationships of chromone derivatives toward mechanism of interaction with, and inhibition of, breast cancer resistance protein ABCG2. J Med Chem 56(24):9849-9860. doi: 10.1021/jm401649j

35. Tiwari AK, Sodani K, Wang SR, Kuang YH, Ashby CR Jr, Chen X, Chan ZS (2009) Nilotinib (AMN107, Tasigna) reverses multidrug resistance by inhibiting the activity of the ABCB1/Pgp and ABCG2/BCRP/MXR transporters. Biochem Pharmacol 78(2):153-161. doi: 10.1016/j.bcp.2009.04.002

36. Hazai E, Bikadi Z (2008) Homology modeling of breast cancer resistance protein (ABCG2). J Struct Biol 162(1):63-74. doi: 10.1016/jsb.2007.12.001

37. Al-Shawi MK, Polar MK, Omote H, Figler RA (2003) Transition state analysis of the coupling of drug transport to ATP hydrolysis by P-glycoprotein. J Biol Chem 278(52):52629-52640 


\section{Figure captions}

Fig. 1. Sequence alignment of human $A B C G$ sub-family members, emphasizing the regions containing the canonical signature/C-motif (marked with a blue line) $(\mathbf{A})$ or a non-canonical C2-sequence that is only present in ABCG2 (marked with red line) (B). Alignment of the linker region containing the non-canonical C2-sequence of human ABCG2 with homologous regions of ABCG2 homologs from various species (C).

Fig. 2. Predictions of disorder within the linker region of human ABCG2 containing the LSGGE C2-sequence at positions 352-356. The arrows show the C2-sequence position.

Fig. 3. Detection and quantification of the $\mathrm{C} 2$-sequence point mutants within the plasma membrane of transfected cells by flow cytometry with the 5D3 surface antibody. Stably-transfected HEK293 cells were incubated with phycoerythrin-labeled 5D3 antibody (blue line) or a negative control antibody (red line) (A). Similarly to wildtype ABCG2, the C2-sequence mutants and the R482G gain-of-function mutant were detected on the cell surface, and quantified by 5D3 fluorescence; the bars represent the mean of median fluorescence (B). HEK293 cells with stable expression of either pcDNA3.1 empty vector, wild-type ABCG2 or the C2-sequence L352A mutant were fixed and immunostained with the ABCG2-specific BXP-21 antibody, and nuclei were counterstained with 4',6-diamidino-2-phenylindole (DAPI) (C) .

Fig. 4. Mutation-induced alterations of ABCG2-mediated drug-efflux activity. HEK293 cells expressing wild-type $A B C G 2$, the different $\mathrm{C} 2$-sequence mutants or the $\mathrm{R}_{482} \mathrm{G}$ gain-of-function mutant were incubated with $20 \mu \mathrm{M}$ mitoxantrone with (blue line) or without (red line) $10 \mu \mathrm{M}$ fumitremorgin $\mathrm{C}$ (FTC) as a specific inhibitor, to generate the 
histograms (A). The efflux was quantified by difference between the two histograms either without further corrections (B) or by taking into account the exact plasma membrane ABCG2 content determined in Fig. 3B (C).

Fig. 5. Mutation-induced sensitization to mitoxantrone toxicity of the growth of multidrug-resistant cells. The different sensitivities to mitoxantrone of transfected HEK293 cells with stable expression of wild-type ABCG2 (in green) or the control pcDNA3.1 empty vector (in magenta) were compared to that of ABCG2 mutants (in black) with the following point mutations: L352A (A), S353A (B), G354A (C) or E356A (D). The cells were cultured on monolayer at day 0 , and increasing concentrations of mitoxantrone were added to cells at day 1 until day 5; they were stained with MTT, and the absorbance read at $570 \mathrm{~nm}$. Data points represent mean values $\pm \operatorname{SD}(n=3)$.

Fig. 6. Differential mutation-induced alterations of basal (A) and substrate-stimulated (B) ATPase activity of ABCG2. (A) Crude membranes from transfected HEK293 cells expressing wild-type ABCG2, or the L352A, S353A or E356A mutant were incubated in the presence or absence of beryllium-fluoride $(0.2 \mathrm{mM}$ beryllium sulfate and 2.5 $\mathrm{mM}$ sodium fluoride) in ATPase assay buffer with increasing ATP concentrations in the absence of substrate drug. (B) The drug-stimulated activity was measured at 5 mM ATP upon addition of $0.25 \mu \mathrm{M}$ nilotinib (grey bars) in comparison to the basal activity without substrate addition (black bars). The results are expressed as mean values \pm standard deviations from three independent experiments.

Fig. 7. Mutation-induced alterations of $A B C G 2$ interaction with different types of inhibitors. The efflux of mitoxantrone by HEK293 cells expressing either wild-type 
ABCG2 (circles) or the S353A mutant (triangles) was measured by flow cytometry as in Fig. 4. Its inhibition by increasing concentrations of Ko143 (A), chromone 1 (B) or nilotinib (C) was plotted as indicated. 
A

\begin{tabular}{|c|c|c|c|c|c|c|c|c|c|c|c|c|c|c|c|c|c|c|c|c|c|c|c|c|c|c|c|c|c|c|c|c|c|c|c|c|c|c|c|c|c|c|c|}
\hline & & & & & & & & & & & & & & & & & & & & & & & & & & & & & & & & & & & & & & & & & & & \\
\hline ABcoit & K & \# & 1 & L & 7 & A & I & a & I L & $1=$ & c & A & $n$ & 1 & $\vec{A}$ & $T$ & a & " & 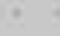 & $=$ & $4 *$ & $x=3$ & 54 & 5 & a & a: & a & A : & F. & $\bar{F}$ & 6 & A & 1 & A & h & F & i & v & $N$ & $N$ & $F$ & $F$ & $\checkmark M$ \\
\hline iecut & 1 & $f$ & 1 & L & $T$ & A & 1 & 6 & 1. $M$ & 45 & 6 & 5 & $\mathrm{H}$ & $T$ & ค & $T$ & $A$ & $=$ & $r$ & * & $=$ & $i$ & 1 & 5 & a & 5 & a & A & k. & A & L & A & 1 & A & $L$ & $\leftleftarrows$ & $L$ & 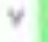 & N & $\mathrm{k}$ & 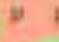 & $p$ & $\checkmark H$ \\
\hline $\cos 2$ & 4 & A & v & 1 & $Q$ & E & t & $a$ & t & $2 k$ & v & A & D & 5 & k. & $v$ & 0 & 7 & $a t$ & F: & $1 \mathrm{~A}$ & A $\pi$ & s. $v$ & s. & 0 & 0 & E & A & $*$ & F & 1. & 7 & 1 & $a$ & $M$ & E & t & 1 & $T$ & c & $\neq$ & $=1$ & 11 \\
\hline Aecra & e & A & $v$ & $M$ & A & $\neq$ & L & 5 & 45 & 54 & $v$ & A & 0 & n & 1 & 1 & $\theta$ & $n$ & $\gamma$ & 5 & $1 \mathrm{a}$ & a $a$ & a 1 & 5 & $T$ & $a$ & E & f & $\pi$ & n & $v$ & 2 & 1 & $A$ & a & a & L & i. & 0 & e & n. & 5. & $\checkmark M$ \\
\hline 2000 & E & D & $v$ & 1 & a & E & L & A & If $\mathrm{F}$ & 80 & c & A & $D$ & 1 & F & $v$ & a & N & $\mathrm{M}$ & $r v$ & $\checkmark F$ & ค $a$ & 1 & s: & $a$ & a & $E$ & F & n. & F. & $v$ & 3 & 1 & a & $v$ & 0 & 4 & 1 & $w$ & $\mathrm{~N}$ & 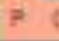 & a & 11 \\
\hline
\end{tabular}

Non-canonical signature

B

\begin{tabular}{|c|c|c|c|c|c|c|c|c|c|c|c|c|c|c|c|c|c|c|c|c|c|c|c|c|c|c|c|c|c|c|c|c|c|c|c|c|c|c|c|c|c|c|c|c|c|c|c|c|}
\hline Ascail & p & I & $\sqrt{i}$ & $\bar{w}$ & $H$ & ศ & N & 5 & c & 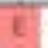 & $v$ & $x$ & $\sqrt{9}$ & $\mathrm{t}$ & * & $n$ & i & K. & 6 & i & $\pi$ & K: & b & 5 & 3 & $\sqrt{3}$ & $M$ & $\widehat{t}$ & a & c & n & 3 & $r$ & 3 & $\approx$ & 3 & c & i & $T$ & 0 & 1 & c. & i & i & $f$ & $\varepsilon$ & $n$ & $t$ \\
\hline NEDO4 & A & P & c & p & e & c & e & p & : & C & v & D. & e & 1 & f & A & $=$ & " & $=$ & $*$ & $=$ & $*$ & $=$ & * & $=$ & * & $=$ & * & $=$ & 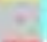 & $\mu$ & $T$ & f & 2 & 7 & 3 & $T$ & t & $T$ & a. & 6 & c: & 1 & L & 5 & c. & ค & $T$ \\
\hline 20 & $v$ & N & 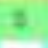 & 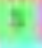 & 1 & $\checkmark$. & K & E & 7 & $R$ & 2 & c) & i & $\mathrm{H}$ & 0 & $i$ & 5 & $\alpha$ & 6 & E & $n$ & $\kappa$ & $x$ & 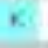 & 1 & $t$ & $v$ & $r$ & $x$ & 1 & 1 & 1 & $v$ & 7 & 7 & $=$ & $r$ & c & $n$ & 9 & 6 & $n$ & & $v$ & 3 & $\kappa$ & $\pi$ & 7 \\
\hline NECOS & 5 & a & $M$ & E & H & L. & E. & I & 1 & e & $M$ & $v$ & e & F & F & $\mathrm{T}$ & 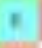 & D. & 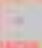 & s. & $=$ & $*$ & $=$ & * & 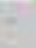 & " & $=$ & " & $=$ & " & $=$ & * & 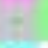 & 3 & e & B. & $v$ & $f$ & 3 & K. & 5 & 9 & $v$ & t & 4 & ค. & ด & v \\
\hline A600 & 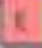 & $t$ & 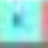 & D) & $i$ & D & 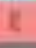 & D. & 7 & c & $v$ & C & 11 & 3. & $\mathrm{v}$ & $T$ & A & $i$ & B & $t$ & $\kappa$ & 17 & 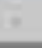 & 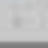 & $=$ & 1 & c & L & n & 3 & 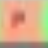 & $t$ & $\kappa$ & $M$ & n & Q & $A$ & $v$ & $\mathrm{c}$ & 0 & 7 & 1 & $T$ & i & 1 & in & $n$ & a \\
\hline
\end{tabular}

C

\begin{tabular}{|c|c|c|c|c|c|c|c|c|c|c|c|c|c|c|c|c|c|c|c|c|c|c|c|c|c|c|c|c|c|c|c|c|c|c|c|c|c|c|c|c|c|c|c|c|c|c|c|c|c|c|c|c|c|}
\hline & $\pi$ & & & & & & & & & 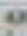 & & & & & en & & & & $=$ & & & & 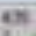 & & & & 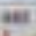 & & & & 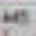 & & & & 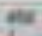 & & & & $=$ & & & & $=$ & & & & e & & & & is & & \\
\hline sous.rues & & & & & & & & s & & & & & & & & & & & & & & & & & & & & & & & & & & & & & & & & & & 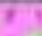 & & & & & & & & & & & \\
\hline gat pues & 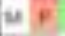 & $t$ & E. & c. & $=$ & 1. & h $=$ & 21 & & & E & 5 & e & 4 & 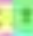 & * & & 5 & 87 & i & c. & c & & 7 & & & 30 & -2 & t & & & 58 & a & z & & & & & $r$ & t & & 6 & & & & & & c) & & & & & \\
\hline 203064 & & & & & & & & 5 & & a & & & & & $5=$ & $\mathrm{P}$ & & & 86 & is & 5 & & & 79 & a & & & r. 1 & & $\mathrm{~s}$ & & $\therefore$ & & & & $1 \pi$ & & & 6 & $=$ & & , & 5. 1 & & & & & & & $+t$ & ' & & \\
\hline tav solw & & 1 & o. & 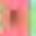 & 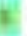 & 1 & $v_{1}$ & f & & $n$ & $\mu$ & $t$ & 1 & $r$. & 1 & D & t & n & 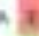 & t & $\mathrm{t}$ & & $1 \mathrm{x}$ & vo & F & 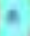 & 18 & c. & 1 & Y & 6 & $\therefore$ & 1 & $p$ & a. & a $\mathrm{A}$ & 1 & 1. $\%$ & $\mathrm{r}$ & 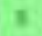 & \pm & , $\mathrm{ts}$ & $c *$ & 2 & 18 & $n$ & $\mathrm{v}$ & 17 & E. & " & , & o & $N 1$ \\
\hline Bronch & & 1 & & & 4 & & & e & & & & & & & $2=$ & & & * & & it & & & & $t \mathrm{~s}$ & & & & $8=3$ & & 5 & & & & & & & & & ro & & & f. & it & & & & & & & & $+\%$ & & \\
\hline cumacec & & r & & & c. & & & E & s & * & a. & is & & - & $a$ & 9 & & & a 8 & & & * & 1 & & & 8 & & at & $T$ & 1 & & $\rightarrow$ & a & - & $x$ & $=7$ & & & 4 & 7 & & , & $1+$ & o & & & v & 1. & & * & 7.9 & 6 & \\
\hline $3000=$ & & 1 & & & 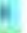 & & & F & & & $\mathrm{s}$ & 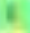 & & & 0 & $a$ & & & $A B$ & is & 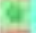 & & & 48 & & & & $8 x$ & & - & & & & e. & & & & & & I & & is & $6 \%$ & & & & & & & & & & \\
\hline Sale 0.6\% & & & $\varepsilon$ & & a & & as 8 & x & & & 4. & & & & 1 & 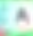 & & h & ac & & & & & 10 & & $n$ & & 73 & & $=$ & 11 & 7. & & I. & c. & 43 & & & & $=t$ & & , 8 & $c a$ & & & & $v$ & & & & 1. & & Nit \\
\hline Whue, ches. & s. & v & $y$ & & $t$ & i. & & . & $r$ & i. & " & 1 & 1 & +1 & 12 & 1 & $\mathrm{~F}$ & e. & $5 *$ & 6 & a & *. & $v F$ & \pm h & - & $x$ & b. & a $*$ & x & $=1$ & 6. 4 & 87 & k & . & 5. & $4 \mathrm{E}$ & 1 & t. & $x^{2}$ & h & a & 1 & E 1 & 0 & $4=$ & 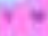 & $v$ & $\mathrm{x}$ & & * & 12 & I. & N \\
\hline tye & & & $\varepsilon$ & & 1 & a. & & 8 & r & i & 4 & 8 & 1 & i & 1 & 0 & If & e. & e. & 1 & & e & 4 & ti & & s & 0 & 6. & & c. 1 & c. & 81 & & * & & 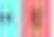 & & & a & $\omega$ & & , & -1 & 0 & & & & & & * & & & \\
\hline Whom MCNDC & & 1 & 1 & & I. & & A 1 & v & r & 1 & 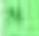 & 4 & $T$ & Y. & . & $f$ & t & * 1 & $A$ & 16 & b & + & E: & 1. & - & - & $A=$ & a $y$ & k & k. & a & c. 5 & & s & $=$ & 41 & & & , 4 & t & 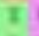 & f & C. $H$ & 3 & & k. & $\%$ & 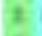 & * & A & 59 & F. & in \\
\hline ath & , & , & 1 & E & 1 & 1. & A $=$ & i & & & s. & 5 & 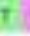 & & , & s & & * & 45 & i & \& & * & 13 & 3. & - & - & as & 6) & 0 & s. & $\mathrm{v}$ & 4. $T$ & $T$ & $v$ & 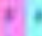 & e 18 & & $1 \mathrm{r}$ & 1 & 7 & & 8 & $F$ & & 4 , & $+*$ & v & & & & & & \\
\hline 40 & & v & 1 & E. & & 13 & & , & r & 1 & a & $=$ & * & i & 10 & $t$ & & & a 8 & i & & $\theta$ & i. & t: & r & ? & 4. & & & c & 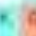 & 6. 7 & & a & 1. & $x=8$ & & & $r$ & $t$ & & of 8 & $\mathrm{Cm}$ & & & & & A & & $=$ & 2 , & & $m=1$ \\
\hline Andes on & & ' & 1 & f & * & $=9$ & a 1 & $f$ & r & 1 & 4 & 1 & $T$ & 1 & 15 & e & $\mathrm{T}$ & - & a. 6 & 1 & fit & $\theta$ & 17 & 1.: & * & $=$ & 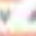 & a $a$ & 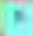 & $=$ & 6 & a 4 & is & A & f & 18 & $t$ & $v=$ & iv & I & 1 & $f$ & $c *$ & 9 & 10 & $h=$ & 1 & 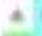 & * & * & 87 & $f \circ$ & $N$ \\
\hline $\overrightarrow{S t}$ & t. & 6 & 1 & & * & 6 & * & 1 & & $\psi$ & 4 & I & $\pi$ & 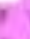 & 17 & 8 & 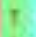 & K. & a 8 & 16 & H & $\theta$ & 5 & 10 & 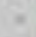 & $*$ & 9 & 58 & & 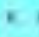 & 50 & $\leqslant 1$ & & $v$ & 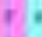 & $<0$ & & $t v$ & 7 & $\pi$ & & , & $6 \%$ & 0 & 6. & $5 \%$ & & $x$ & & n & 3, & & $w$ \\
\hline For & & if & 1 & 1 & * & & ${ }_{n}=$ & 1 & & & 4 & $z$ & $a$ & f & 1. & $t$ & & & a) 8 & & & 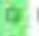 & & 20 & & 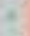 & 30 & 21 & & 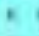 & 5 & 01 & $t$ & $v$ & 1. & $<1$ & 1 & & $6 \pi$ & $6 x$ & a & , & $c=$ & 0 & & & & 1 & & * & 20 & 18 & w \\
\hline & t. & if & 1 & 18 & c. & 2 & 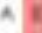 & 1 & & 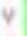 & 4 & $\mathrm{n}$ & I & 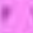 & 1 & 4 & r & 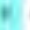 & a 8 & it & 94 & 9. & 13 & I: & $*$ & +7 & 4 & 08 & x & 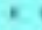 & 10 & $\approx 1$ & t & $v$ & 10 & 56 & 1 & 17 & 7 & 7 & & , & $c=$ & 2 & 10 & f $v$ & $\checkmark$ & 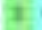 & & * & , & & $N$ \\
\hline 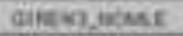 & & 6 & 1 & E: & E & & $k=$ & 1 & v & 1 & 4 & i & $a$ & 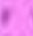 & 6. & $E$ & $t$ & $k$ & a 1 & t & $H$ & $\theta$ & 67 & $\alpha=$ & , & * & 47 & 16 & & $\therefore$ & 6. & $\approx 1$ & t & $v$ & $r$ & 6. $t$ & 1 & $E v$ & 6 & $t$ & & $f$ & 6 & 2 & 60 & $n=$ & $v$ & 1. & & " & 1, & $2 \%$ & $k$ \\
\hline 69 & & i & $y$ & & *. & 2 & $A=$ & 1 & * & v & D. & 1 & 8 & $f$ & 1, & $t$ & $t$ & k. & A 1 & i & $M$ & $\theta$ & 17 & 10 & 2 & ets & 9 & $5 x$ & $x^{2}+2$ & \& & x. & $\theta 1$ & T & $v$ & $\neq$ & 61 & & & 11 & 1. & & $=$ & $c$ & 9 & 1. & A * * & $v$ & 1 & & . & 17 & $F x$ & 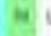 \\
\hline 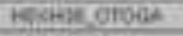 & & i & 1 & 1 & c. & 14 & $\mathrm{~A}$ & , & v & $\mathrm{v}$ & 4 & 1 & 2 & 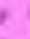 & 1. & 1 & $t$ & . & a 1 & is & 8 & 0 & 1 & 12 & 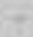 & $\Rightarrow$ & 4. & 1. & & n & $x=$ & $x t$ & $r$ & A & is & 18 & & $A=$ & $1 \mathrm{~A}$ & It & & , & $c=$ & 0 & is & $n=$ & $v$ & 1 & & 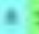 & 3 , & $f^{\prime}$ & $N$ \\
\hline 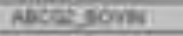 & & is & 1 & E & . & 1. & 21 & 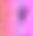 & 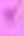 & 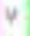 & 4. & 8 & 3 & 1 & 1. & $t$ & 1 & 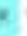 & $\mathrm{v}$ & i & 8 & 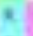 & 8 & $3=$ & 2 &. & 4 & $b 0$ & $n$ & . & . & 2. & In & $y$ & 7 & ${ }^{\circ}, 8$ & $\mathrm{v}$ & 1. & 14 & 1 & 1 & , & $c=$ & 0 & i. & c. & 1 & 4 & & * & 2, & $1 \%$ & $*$ \\
\hline 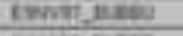 & & i & 1 & I & 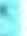 & 1. & $k=$ & t & 7 & 1 & 4 & $t$ & 2 & 4 & 1. & $f$ & t & N. & $v$ & 1 & $\mathrm{e}$ & *. & +1 & 10 & t & 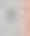 & 81 & 50 & s & 5 & N & $\approx 1$ & 1 & $t$ & $\%$ & 4 & $v$ & t. & is & 1 & & , & $c \pi$ & 2 & & $k=$ & 1 & 1 & & A & 1, & & \\
\hline cooseng & & 6 & 1 & 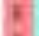 & *. & 4 & $A$ & 1 & F & 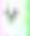 & 4 & 5 & 3 & 8 & 1. & 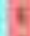 & 1 & * & 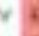 & i & $E$ & $*$ & 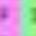 & I. & $=$ & $=$ & 57 & 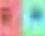 & 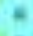 & . & $\therefore$ & $\angle 1$ & 3 & 3 & 7 & $5 \times$ & 1 & 14 & I & 1 & I & " & $c=$ & $\partial$ & 6 & $k$ & , & 3 & & * & 37 & 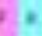 & $\mathbb{N}$ \\
\hline 10 & & is & 1 & If & א. & 4 & $A \geq$ & I & $y$ & $v$ & 4 & 2 & $\pi$ & $\Rightarrow$ & 1. & t & $t$ & k. & $r$ & it & $\theta$ & $*$ & r. 1 & $7=$ & t & $*$ & 9 & 10 & A & 6 & 5. & $\therefore 2$ & 17 & $x$ & $\gamma$ & $F_{1}$ & 1 & T. & 14 & 1 & $n$ & s. & $c=$ & 0 & 67 & $5=$ & 1 & 1 & & 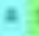 & $2 \%$ & 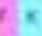 & s. \\
\hline sace & & & & $=$ & 4 & 11 & ${ }^{n} \mathrm{~A}$ & I' & & & 4 & 8 & & 1 & 1. & if & & (.) & $\mathrm{ve}$ & 1 & & a & 6 & 8. & - & 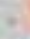 & 4. & 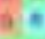 & &. & a. & 67 & 1 & $v$ & $\mathrm{x}$ & of & $v$ & $1=$ & 17 & t & 8 & s & $c=$ & 8 & 1 & $n$ & 1 & 1 & & * & 17 & , & $*$ \\
\hline 00 & & $f$ & 1 & E & 5 & 1. & $A z$ & 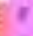 & 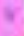 & 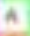 & $N$ & 1 & & 1 & 21 & , & & 4 & $E:$ & 16 & 8 & & 6 & $8=$ & $\theta$ & 4 & 5 & 10 & $x$ & F & $\Rightarrow$ & $<1$ & 1 & A & 1 & 58 & & & $6 *$ & $\pi$ & I & II & 60 & $\theta$ & 64 & $5=$ & 1 & 1 & & $\Rightarrow$ & 11 & I\% & 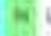 \\
\hline & & I & t & 1 & . & 4 & $A$ & $\because$ & & 9 & 4 & 8 & $T$ & 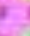 & $5-1$ & 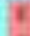 & & 5 & $a$ & 6 & & $\theta$ & & 2. & 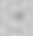 & & 4 & $A=$ & & 8 & 5. & 62 & & $A$ & F, & 61 & & & IV & t & & 6 & $5 \%$ & 2 & & & & 1 & & 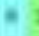 & , & & 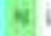 \\
\hline fats $>>$ & & 1 & 1 & $y$ & c & 4. & $x_{1}$ & 1 & & A & 4 & I & $\tau$ & 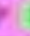 & 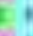 & I & $t$ & K. & 12 & is & & 0 & 4 & I. & 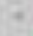 & & 4 & 80 & $x$ & E & 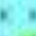 & $\Leftrightarrow I$ & $t$ & A & t. & 616 & & & $1 / 2$ & $t$ & & 1 & $c=$ & 0 & 4 & E & $v$ & $t$ & & * & 21 & $1 \%$ & $N$ \\
\hline keren 1 atw & & c & a & is & a. & 2 & A & E & 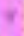 & c. & 4 & 2 & A & . & r. & I & t & e. & vo & it & & 1 & 13 & $\mathrm{t}=$ & . & $*$ & 13 & 5 & s & c. & e. 1 & t. & $=$ & 1 & , & 12 & 1 & t & I & 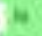 & 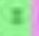 & f. & $1=$ & 0 & & c. $=$ & v & 3 & & * & 1, & 10 & w. \\
\hline & & & y & it & 4 & 1 & 12 & 20 & & & 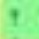 & * & & 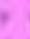 & 18 & t & & s. & 28 & E & & & $u$ & 10 & . & & 6 & 6. & & v & * 1 & in & & $F$ & + & $=0$ & , & & 14 & 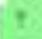 & 1 & - & $i=$ & 0 & & $k=$ & v & e & & a & 1. & in & $n$ \\
\hline 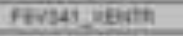 & & & & & & is 3 & & & & 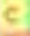 & & $=$ & 2 & & 6 & 1 & & & & & & & & & & & & & & & & & 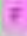 & & & is & & & ra & T & & & $i s$ & $?$ & & E* & $v$ & 5 & c. & 23 & 37 & E. & N \\
\hline & 4 & & & $\nabla$ & & 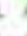 & $*$ & & & & 5 & & & & & & t & & A & & & & & & & & & & & & & - & & & $-\infty$ & $\cdots$ & & & & 2 & & & a. $\mathrm{v}$ & & 6 & & 4 & $t$ & & 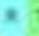 & & & 5 \\
\hline & & & & 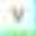 & & & & te & & & & & & & & 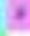 & t & & A & & A & * & t & $t$ & & & $=$ & 4. & & & 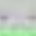 & - & & & 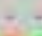 & = & & & & 3 & & & A $\mathrm{v}$ & 2 & & $y t$ & 1 & $t$ & & $*$ & w & a. & e \\
\hline AnE wath & & & & & & & & & & & & & & & & & & & & & & & & & & & & & & & & & & & & & & & $7 \times$ & & & & & & & 18 & & & & & & & M. \\
\hline 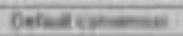 & & & & & & & & & & & & & & & & & & & & & & & & & & & & & & & & & & & & & & & - & & & & ta & & & n. & $y$ & & & & 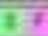 & 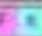 & \\
\hline
\end{tabular}



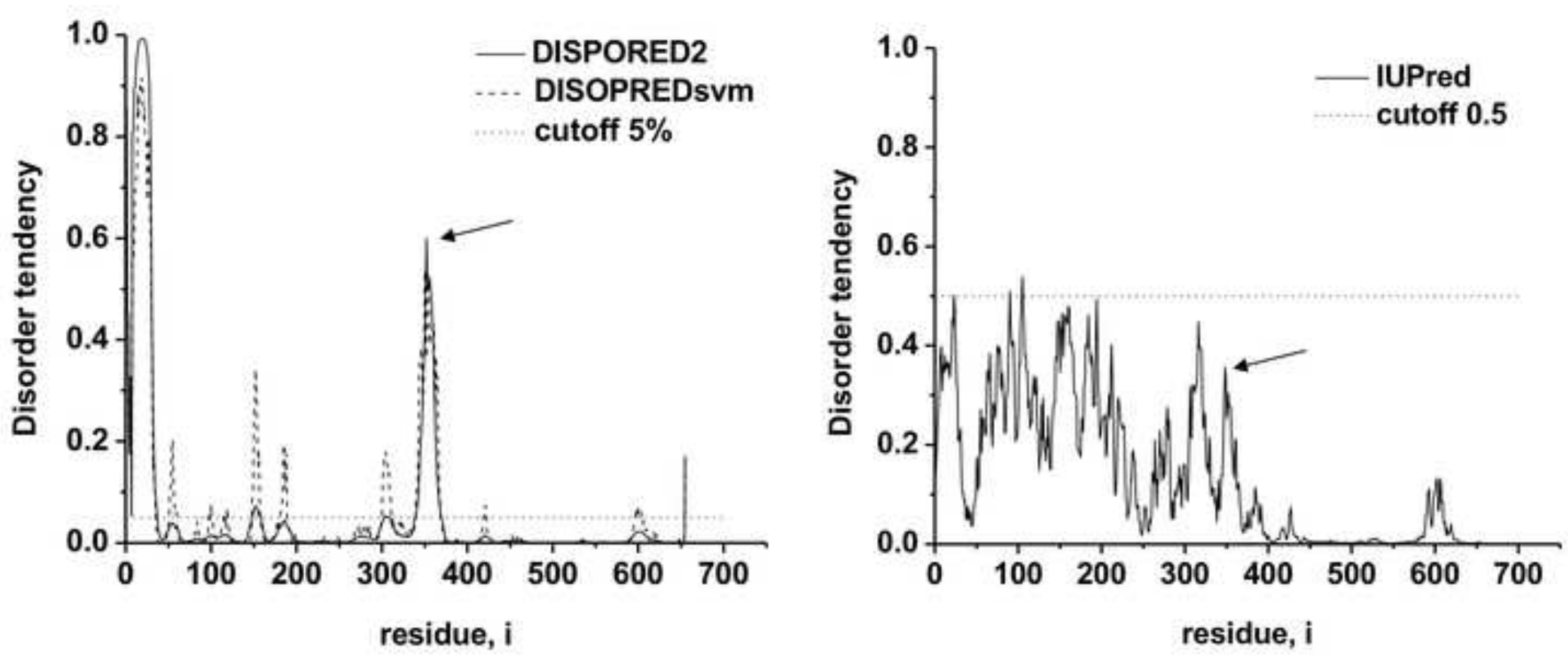
A
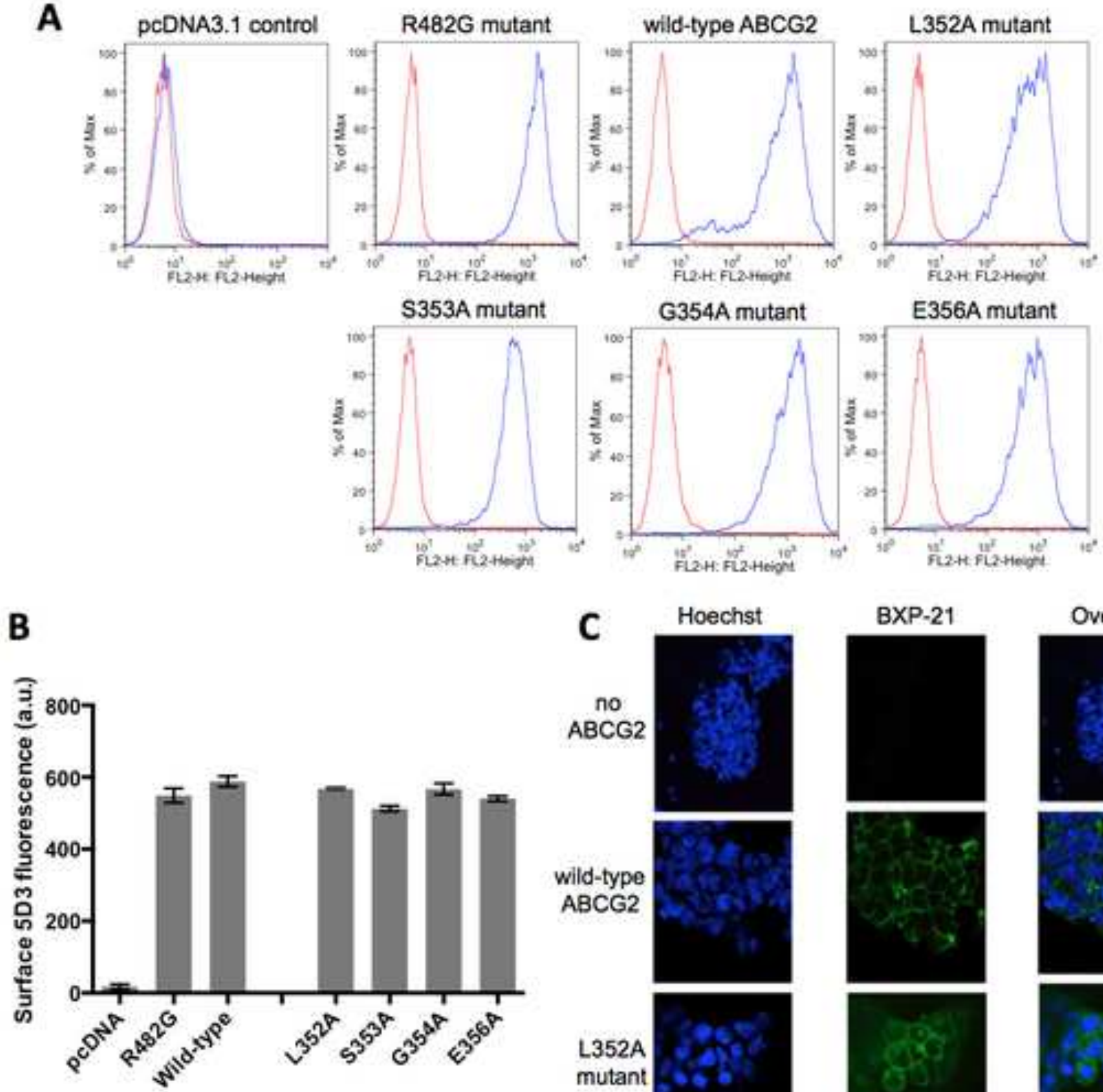

C

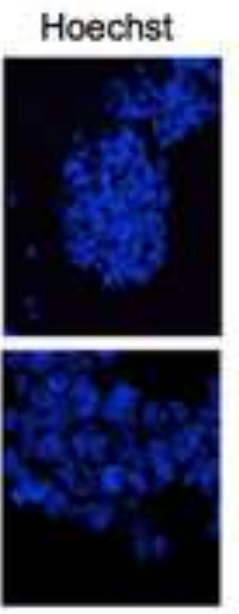

BXP-21
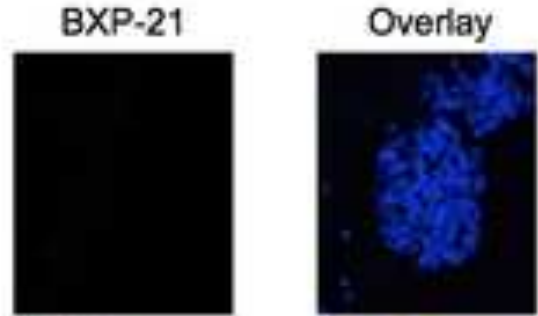

wild-type

ABCG2
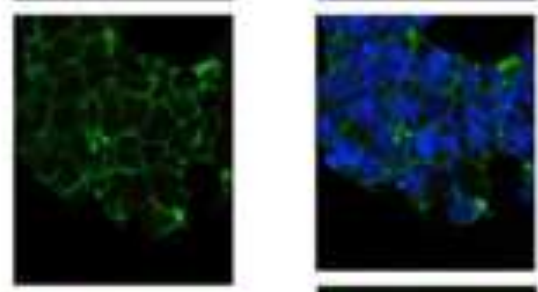

L352A
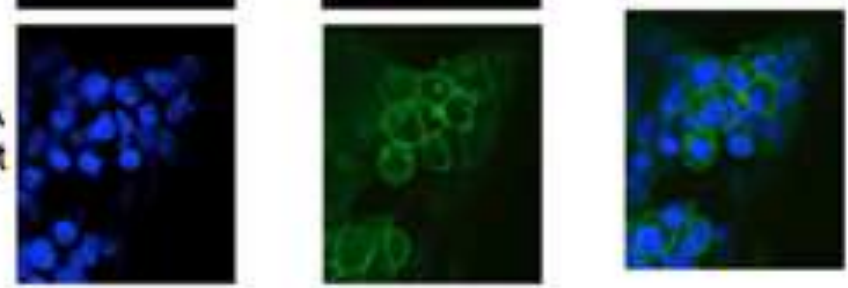

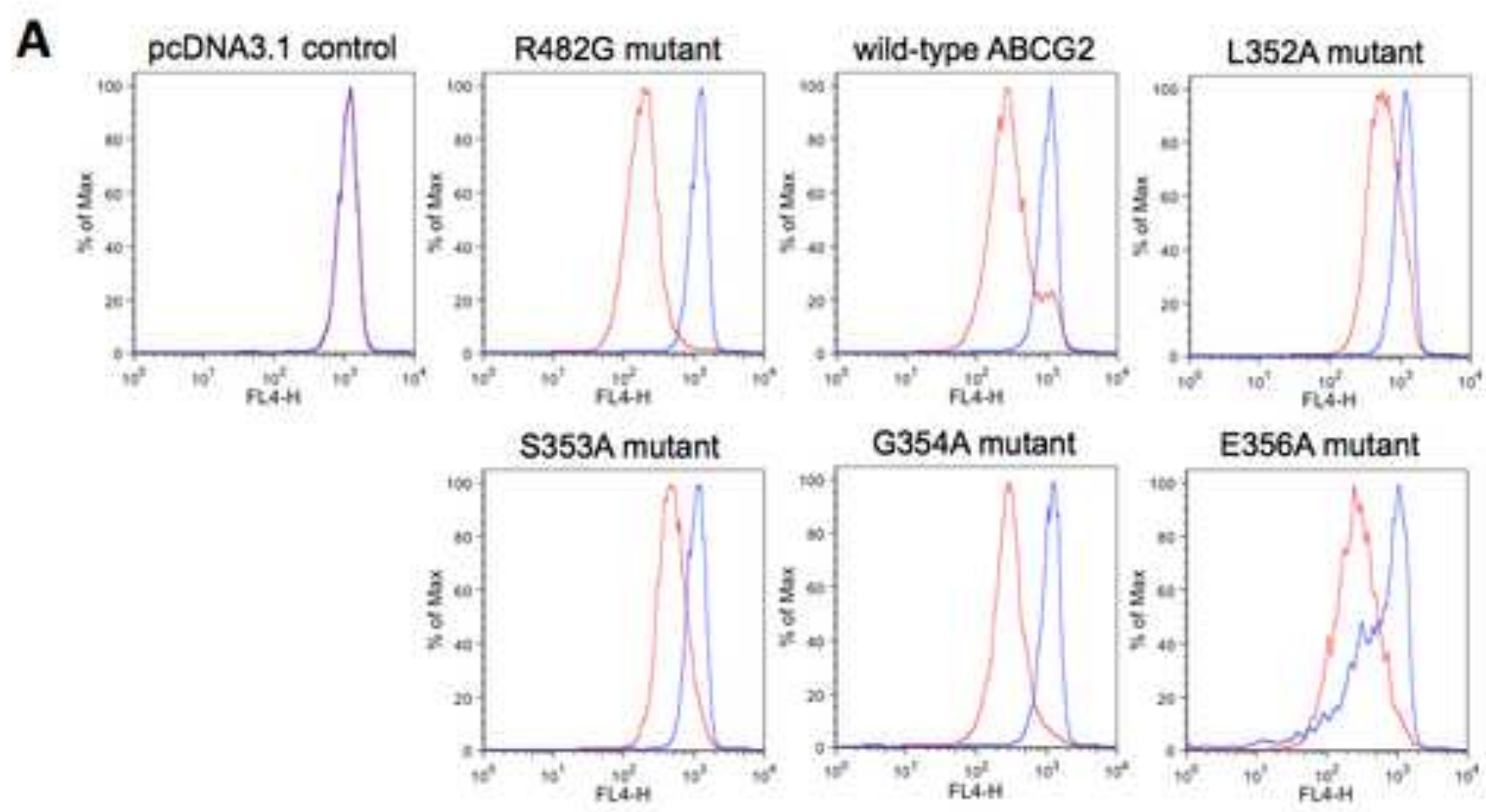

B

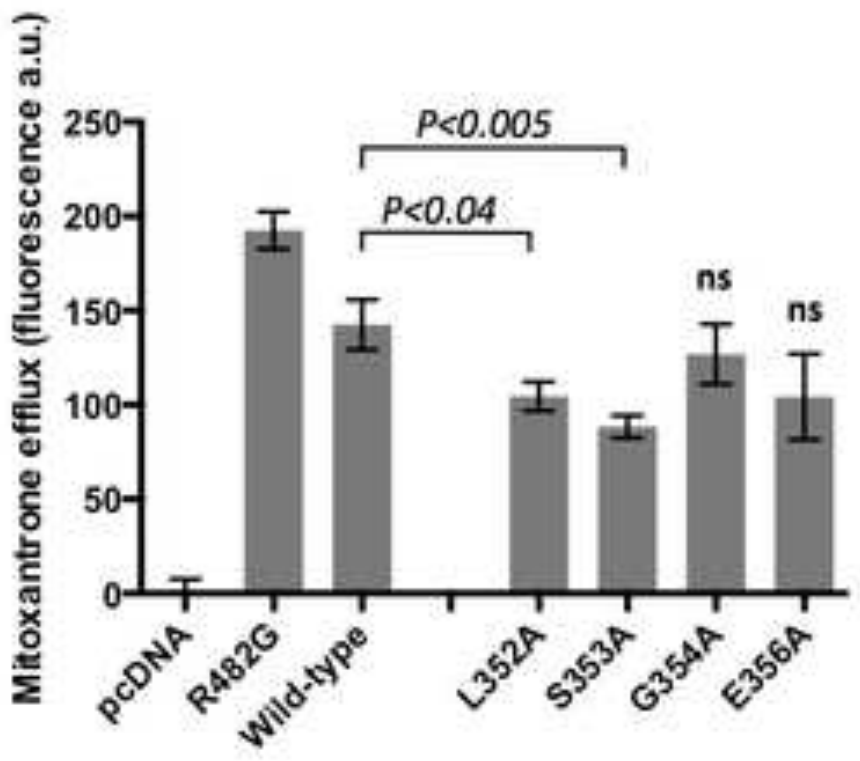

C

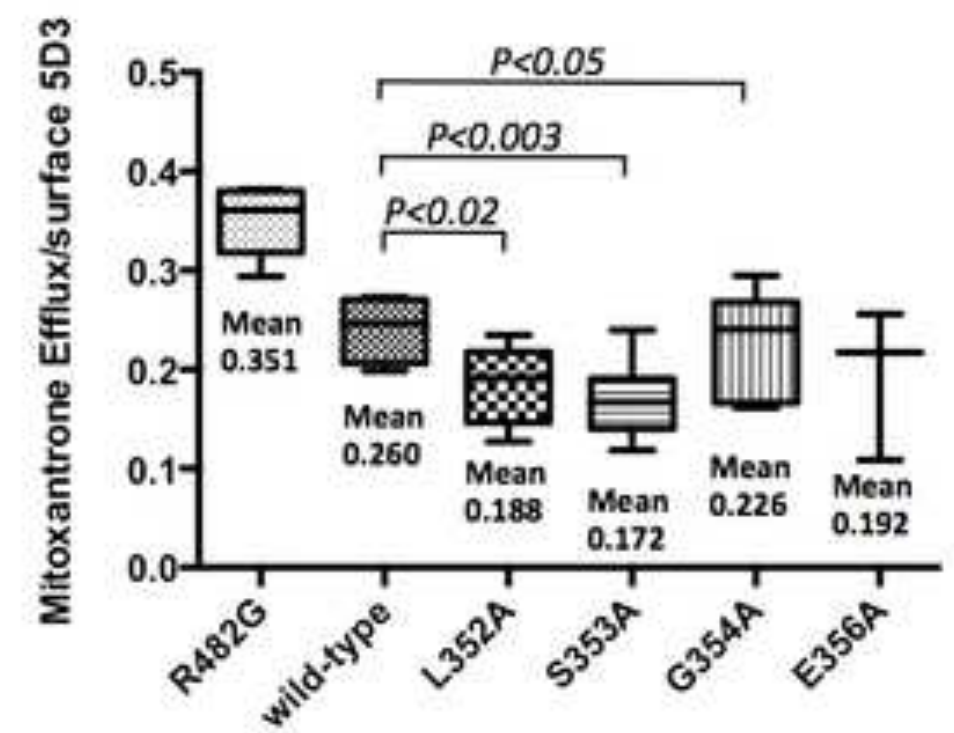


A

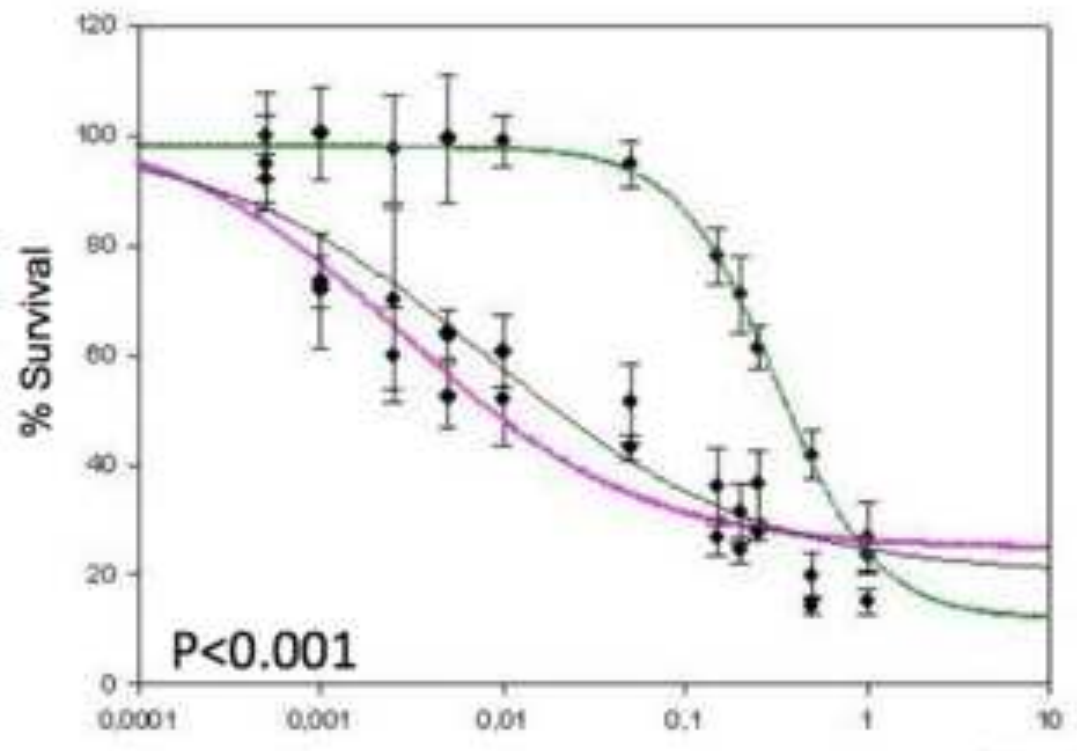

B

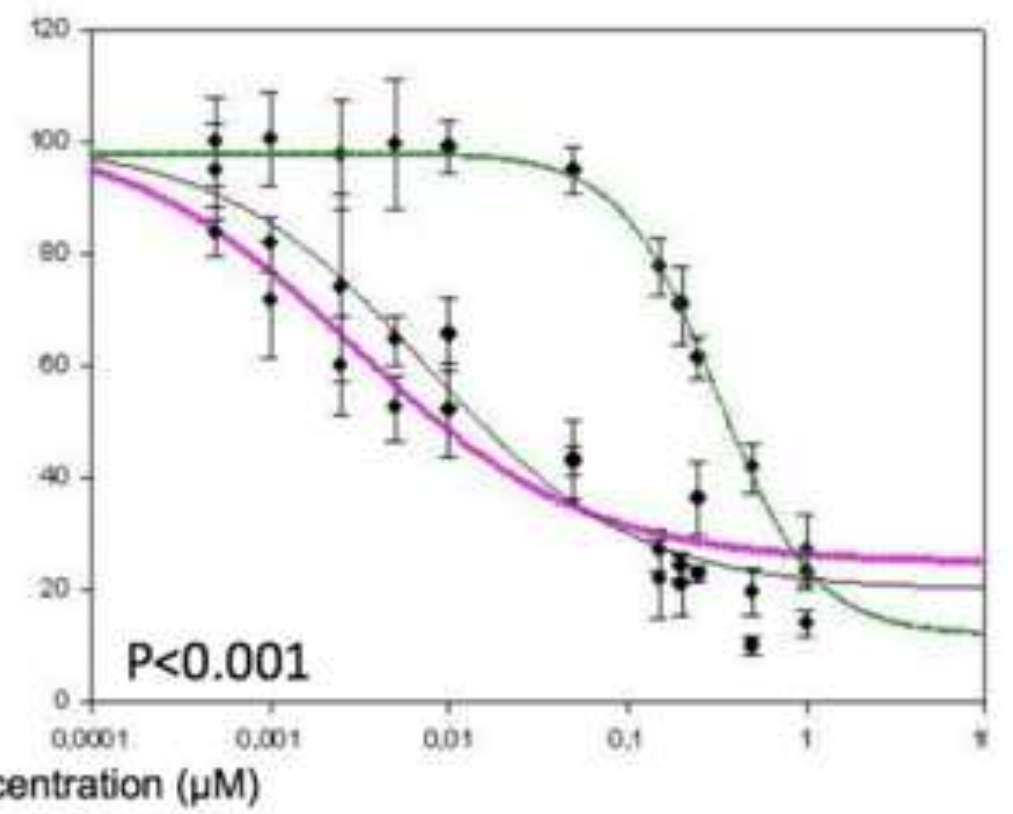

C

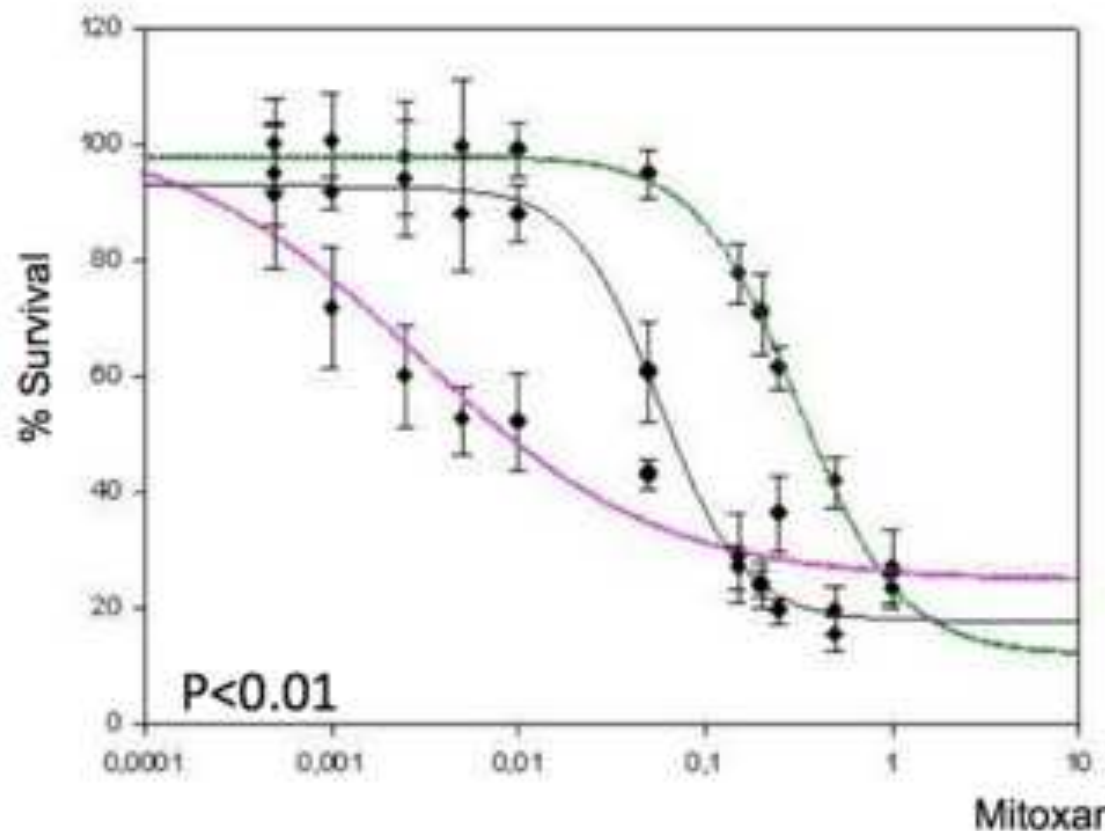

D

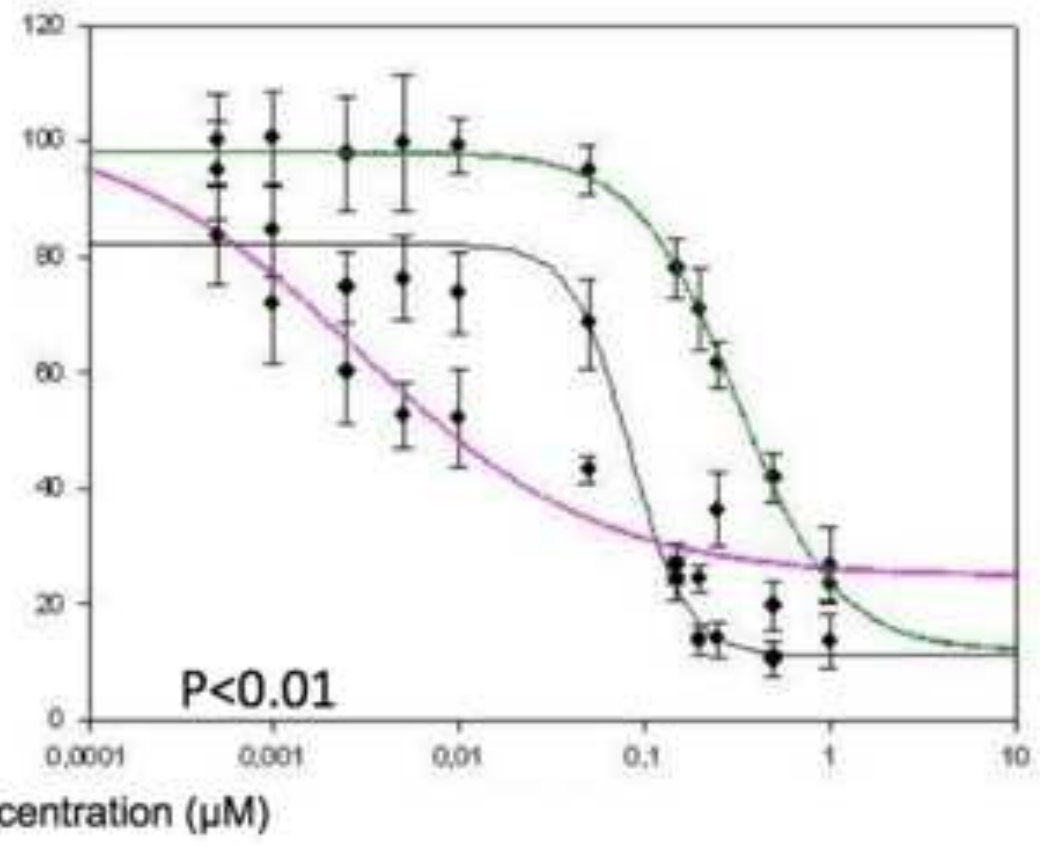

Mitoxantrone concentration ( $\mu \mathrm{M}$ )

Mitoxantrone concentration ( $\mu \mathrm{M})$ 
A

\begin{tabular}{|c|c|c|c|c|}
\hline & Wild-type & L352A & S353A & E356A \\
& ABCG2 & mutant & mutant & mutant \\
\hline $\mathrm{V}_{\max }$ (nmol Pi/ & 14.6 & 11.3 & 10.2 & 11.1 \\
mg protein.min) & 14.4 & 0.15 & 0.06 & 0.46 \\
\hline $\mathrm{K}_{m} \mathrm{ATP}(\mathrm{mM})$ & 0.45 & & & \\
\hline
\end{tabular}

\section{B}
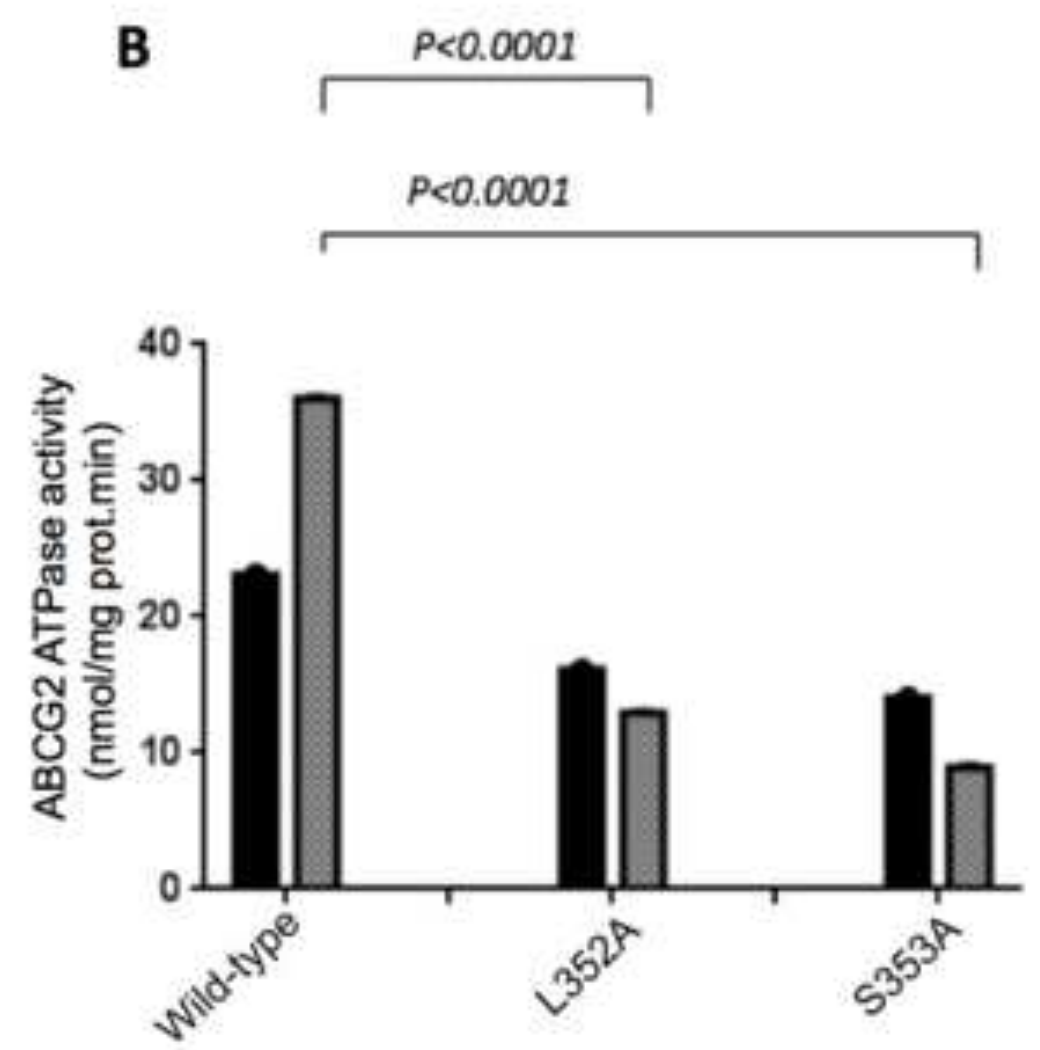
A

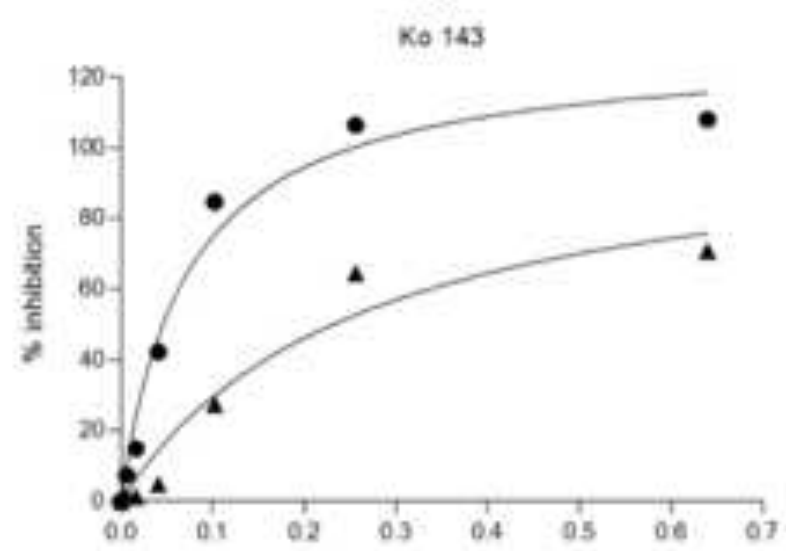

B

Chomone 1

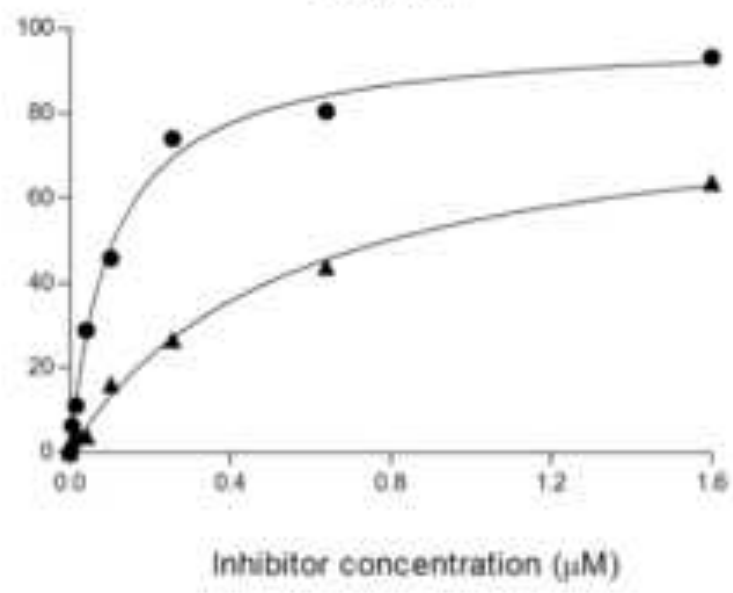

Niotinits

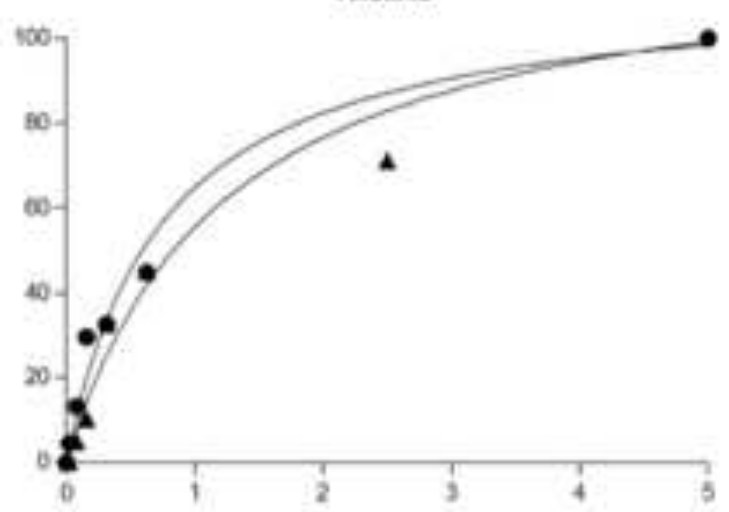


Click here to access/download Supplementary Material Answers_to_Reviewers.doc 